\title{
Monitoring urban dynamics: the case of the Metropolitan area of Central Tuscany
}

\author{
R. M. Pulselli, P. Romano, V. Niccolucci \& N. Marchettini \\ Ecodynamics Group - Dept. of Chemistry, University of Siena, Italy
}

\begin{abstract}
How can human activity be monitored in space and time? To answer this question it is necessary to develop new tools for investigating dynamic phenomena in territorial systems.

In this study, we elaborated data, routinely registered by a mobile telecommunication company, in order to develop a monitoring technique of population density, assuming that the intensity of mobile-phone activity in a cell (a covered area around an antenna) is directly proportional to the presence of people. In this paper, we present an application of this technique to the Metropolitan Area of Central Tuscany (Italy).

Results were represented dynamically in sequences of maps that provide a qualitative description of population behaviour in the form of spatial patterns evolving in time. A quantitative description was also provided based on the measurement of cell-phone calls intensity within given areas. Data elaborating and geo-processing allowed for combining spatial and time-series analysis and understanding the general functioning of a comprehensive geo-demographic system.

The methodology discussed here intends to give a contribution towards the implementation of bottom-up procedures in order to provide information on use and development of urban metropolitan systems in time and to improve our capacity to handle dynamic processes and thus inform planning practices and future developmental strategies.
\end{abstract}

Keywords: cell-phones, mobile telecommunication technology, geo-processing, urban dynamics. 


\section{Introduction}

Kinetics is the science that studies movement or, in general, the circulation of mass. Kinetic theory was born to explain the essential characteristics of matter, based on the hypothesis that it is made up of countless small particles in movement. In the common application to gases, this theory allows us to calculate the macroscopic behaviour of gas based on the microscopic properties (velocity, kinetic energy, etc.) of the molecules it is composed of. Chemical kinetics studies the variations of the concentration of reagents and products in time and can be established experimentally by observing, with appropriate tools, processes and chemical reactions [1].

In this paper, a monitoring technique was developed in order to investigate dynamics in urban systems, focussing on population behaviour and its variation in time. These dynamic processes, obtained through the elaboration of statistical data gathered through the technology of mobile telecommunications, were represented as a comprehensive whole in which individuals operate as the constitutive elements of a complex system and determine its spatial and temporal configuration. A bottom-up data collection was thus developed and data elaboration through geo-processing allowed us to develop a study approach for understanding the dynamic evolutionary properties of urban systems and to reveal their general functioning [2]. This is at least an investigation of kinetic processes in urban systems.

\section{Materials and methods: investigating kinetics in Tuscan metropolitan area}

A research aimed at developing tools for urban dynamics monitoring was first developed at the Massachusetts Institute of Technology in 2004 [3] and then elaborated by the researchers of ecodynamics group in Siena [4-7]. The monitoring technique was studied to observe the variations of population density in real time, and analyze its movements and dynamics in relation to the physical space of the city.

This paper presents results from a research project, namely MoTo (Mobile Toscana) funded by the General Directorate of Territorial and Environmental Policies of the Tuscan Region. Through the systematic collection of statistical data and its aggregated processing and geo-processing, MoTo developed a procedure for obtaining a real measure of population density variable in space and time. In particular, MoTo elaborated data registered by a mobile telephone company in a vast area, which is relative to the position-direction and to the activity of the antennas that transmit the signal to cell-phones. In this study, we assumed that, with a close approximation (in Italy in 2006, the percentage of stable users was registered at between $80 \%$ and $85 \%$ of the population - EITO 2007), the intensity of cell-phone activity is proportional to the number of users present in a given area, and that the variations in activity correspond proportionally to the variations in population density. 
Data was registered by each individual antenna that transmits its signal over a defined area, called a cell. Coordinates and directions of the antennas allowed for the detection of the structure of the mobile phone network throughout the territory, and of the dimension-position of the single cells (Figure 1).
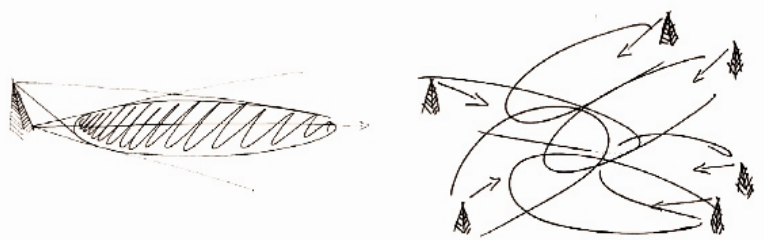

Figure 1: Scheme of antennas and cells.

Activity data from the antennas, referred to the relative cells, was processed and connected to single spatial units identified by a reference grid $(500 \times 500 \mathrm{~m})$. The representation of data in the grid allowed us to visualize the intensity of activity registered in the form of an overall spatial configuration (Figure 2). The grid is extended over an area of $45 \times 35 \mathrm{~km}$. The $90 \times 70$ pixels identify a set of 6300 pixels/area defined as the minimum spatial unit of reference for the processing of the information on a cartographic basis.
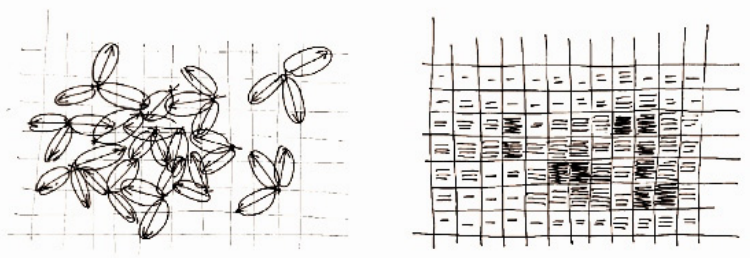

Figure 2: Scheme of spatial elaboration of data in a grid of $500 \times 500 \mathrm{~m}$ pixels.

The minimum spatial units have been aggregated into areas corresponding to urban zones with specific characteristics regarding physical conformation, structures, and land use. The correlation between aggregate spatial units and ISTAT census zones allowed us to obtain results that are comparable with the Territorial Information System (SIT) of the Tuscany Region (basic cartography and ISTAT processing).

The activity of the antennas (number of calls) was registered at hourly intervals per cell sectors in subsequent segments of 550 meters along the direction of propagation of the signal. The study area is a portion of the territory of the Region of Tuscany, which includes the metropolitan area of central Tuscany. This includes the Florence-Prato-Pistoia plain and the zone of Empoli and extends over $1575 \mathrm{~km}^{2}(45 \times 35 \mathrm{~km})$, with over 1,200,000 inhabitants. 
Sequences of patterns with varying intensities dynamically showed the population density and its variation in space and time.

\section{Results and discussion}

Data geo-processing allowed us for representing dynamically the activity of population living and working in the area under study, that is for representing the geo-demographic system of the metropolitan area in Tuscany.

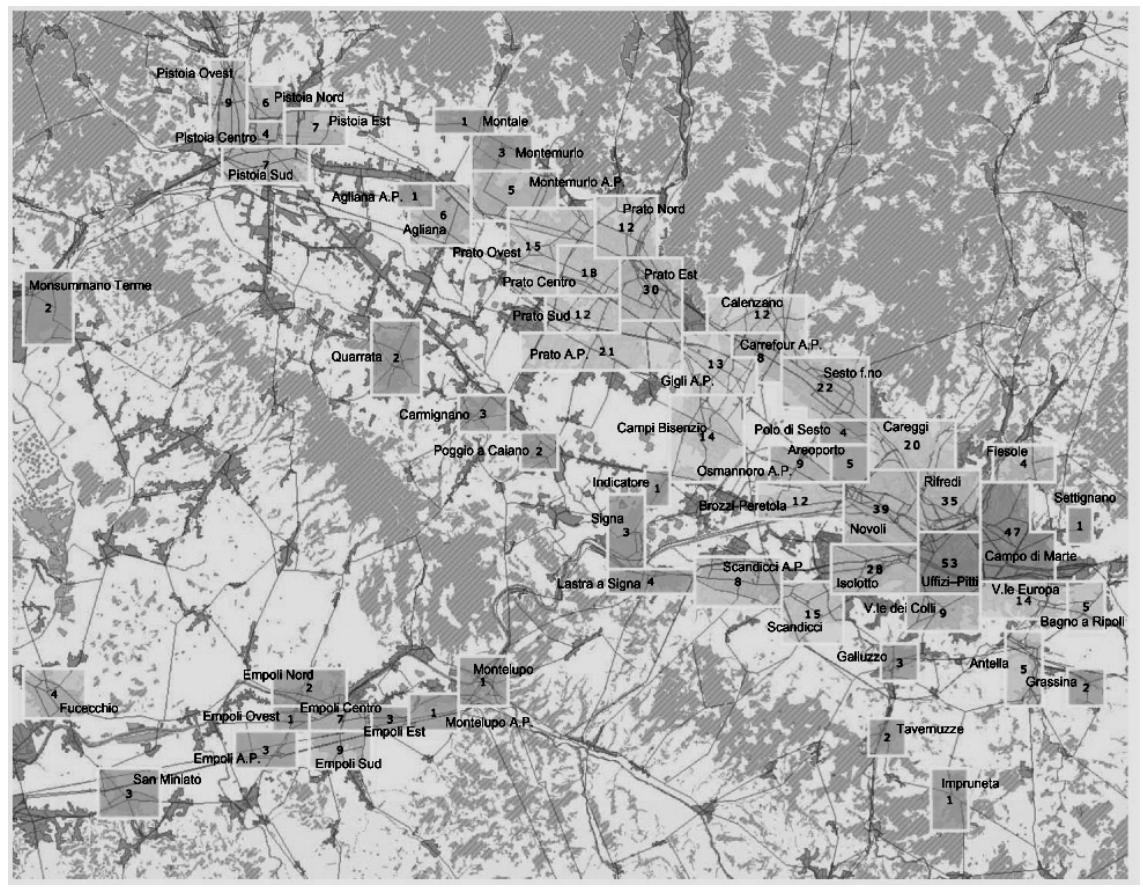

Figure 3: Map of aggregate spatial units in the metropolitan area of Central Tuscany. Values are given in thousandths.

In particular, sequences of maps were elaborated in order to show the evolution of daily activity (total 24 hours) in twelve months or the hourly activity in the 24 hours of a working day comparing different seasons. Maps show cellphones activity in a colour-scale through minimum spatial units. This is also represented through values that correspond to the portion of activity in each aggregated spatial unit given in thousandth relative to the total activity in the area under study $(45 \times 35 \mathrm{~km})$. Spatial patterns and values allowed for representing both qualitative and quantitative aspects of the human activity in the metropolitan area. In Figure 3 a synthetic map shows results in a typical working day. 
The highest intensity is registered in the centre of Florence (aggregate spatial units: Uffizi-Pitti, Campo di Marte, Rifredi, Novoli), and in the areas of Sesto Fioretino and Prato (including the productive area Prato A.P.). A significant concentration is also shown in the areas of Pistoia and Empoli.

In the following diagram (Figure 4), the intensity of cell-phone activity is shown in twelve months. Values refer to the total activity in 24 hours of a working day. It is clear that the activity is almost constant during the year except for June in which it increases, probably due to the incoming of tourists at the beginning of summer season, and in July and August in which it decreases, due to the summer break, until September.

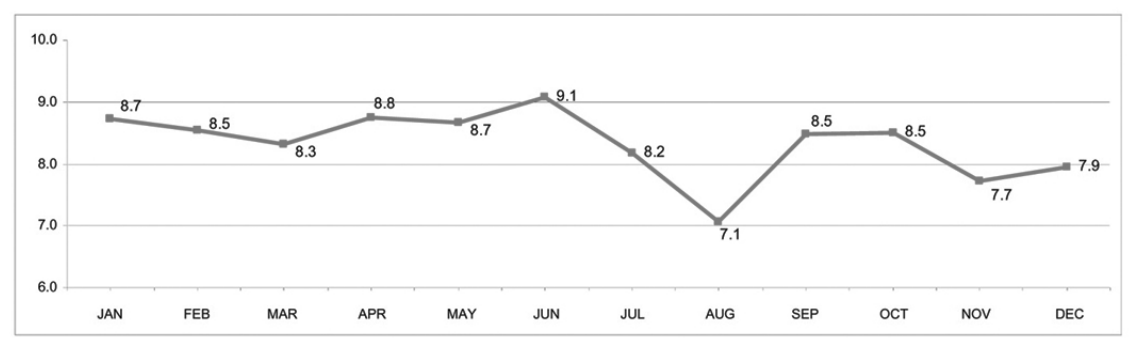

Figure 4: Diagram of total 24hours cell-phone activity during a year (2009) in the metropolitan area of Central Tuscany.
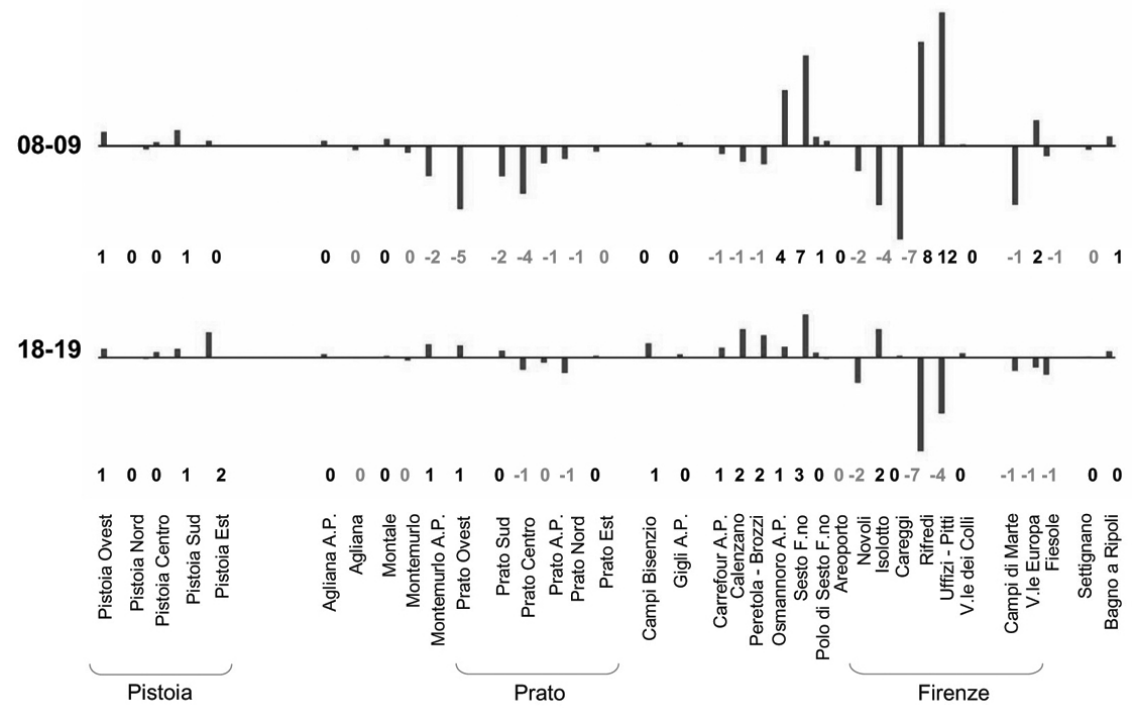

Figure 5: Diagram of cell-phone activity variation in time intervals (8-9 am and 6-7 pm) in the metropolitan area of Central Tuscany. 
Other results show the mode of use in time of specific aggregate spatial units. In particular, among others, two aspects deal with daily life in given areas.

In Figure 5, the two diagrams show the variation of activity in the time intervals from 8 to 9 am and from 6 to $7 \mathrm{pm}$, respectively. The first diagram, that represents variation in early morning, shows an increase of activity in the city centres of Florence, Sesto Fiorentino and Pistoia, as well as in some of the productive areas (Osmannoro, Polo di Sesto, Gigli). The second diagram, that represents variation in the late afternoon, shows a decrease of activity in the areas above and an increase in residential districts in the metropolitan areas, especially around Florence (i.e. Calenziano, Campi Bisenzio, Prato Ovest, Bagno a Ripoli).

In Figure 6, curves show the intensity of activity in 24 hours of a working day in five productive areas corresponding to the aggregated spatial units represented in the map in the right side. Results highlighted here the increase of activity during the early morning during which there are workers incoming, while activity progressively decreases during the afternoon. This shows how working hours effect the intensity of cell-phone activity, for example in the lunch break (from 1 to $2 \mathrm{pm}$ ) or after works.

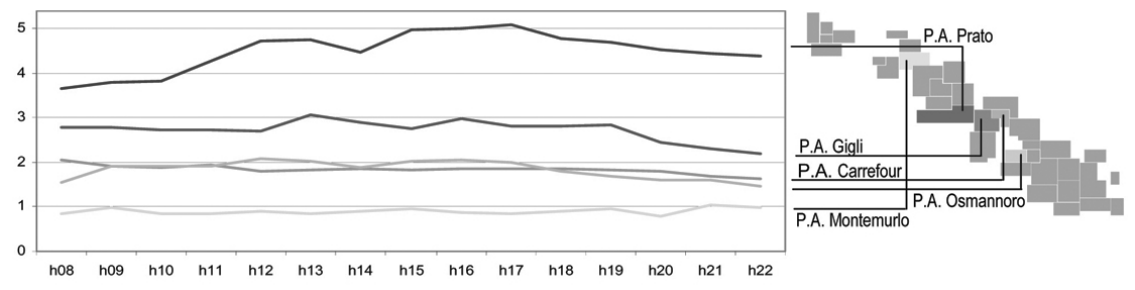

Figure 6: Time series analysis of human activity in five productive areas in the metropolitan area of Central Tuscany during 24 hours of a working day.

\section{Conclusion}

Cell-phone traffic data was elaborated for monitoring population density in time. Patterns of cell-phone activity, with variable intensity, were observed in order to visualize time modes of urban space use and to understand when and how long people use urban space. Results were obtained concerning a) the experimentation of a methodology for monitoring urban dynamics and b) the application to a case study, the Metropolitan Area of Central Tuscany.

a) On the one hand, this study developed a monitoring technique for analysing the geo-demographic system in a vast region based on cell-phone calls. Information provided concerns the methodology used and the procedures of data collection and its statistical elaboration, geo-processing and spatial approximation, spatial analysis and time series analysis. This work would 
contribute for elaborating bottom-up procedures for monitoring human activity in urban areas and providing information on their dynamics.

b) On the other hand, this technique was applied to a region in order to detect the general functioning of a wide metropolitan area and show the comprehensive behaviour of its population in time, for example in a sequence of working days. Data was elaborated relative to minimal spatial units in order to give a qualitative description of spatial phenomena in time through patch dynamics. Quantitative information was also provided relative to aggregated spatial units, in order to give a measure of the intensity of use of urban space by population. Both qualitative and quantitative descriptions were used to show the variation of activity in time and to classify different parts of the system based not only on their structure or function but also on their time of use.

\section{Acknowledgements}

The study of the Metropolitan Area in Central Tuscany, namely MoTo project, was selected from the competition for research and innovation in the territorial and environmental field, and funded by the General Directorate of Territorial and Environmental Policies of the Tuscan Region. We thank the team of the mobile telecommunication company Telecom Italia for collaborating with us and making this project possible.

\section{References}

[1] Pulselli R M, Morandi F, Tiezzi E. Integrating thermodynamics and kinetics of urban systems for regional studies. (Eds.) Brebbia C A., Hernandez S, Tiezzi E. The Sustainable City VI, Urban Regeneration and Sustainability. WIT Press. Southampton, UK 2010 103-111.

[2] Pulselli R M, Tiezzi E. City Out of Chaos. Urban Self-organization and Sustainability. WIT Press, Southampton UK 2009. (Foreword by Paolo Portoghesi).

[3] C. Ratti, R.M. Pulselli, S. Williams, D. Frenchman. Mobile Landscapes: using location data from cell phones for urban analysis. Environment and Planning B: Planning and Design 33(5) 727-748 2006.

[4] Pulselli R M, Ratti C, Tiezzi E. City out of chaos: social patterns and organization in urban systems. International Journal of Ecodynamics 1(2) 2006 125-134.

[5] Bastianoni S, Pulselli R M, Romano P, Pulselli F M. Dynamics and evolution of urban patterns: the evidence of the Mobile Landscapes project. (Ed.) Brebbia C A. Design \& Nature IV-Comparing Design in Nature with Science and Engineering. WIT Press. Southampton, UK 2008 253-260.

[6] Pulselli R M, Romano P, Ratti C, Tiezzi E. Computing urban mobile landscapes through monitoring population density based on cell-phone chatting. International Journal of Design \& Nature and Ecodynamics 3(2) 2008 121-134. 
164 Ecosystems and Sustainable Development VIII

[7] Marchettini N, Pulselli R M, Tiezzi E.B.P. An innovative survey of urban systems dynamics: the evidence of the MoTo project. (Eds.) Brebbia C A, Hernandez S, Tiezzi E. The Sustainable City VI, Urban Regeneration and Sustainability. WITpress. Southampton, UK 2010 97-102. 